\title{
Effect of Flap Fixation Technique in Modified Radical Mastectomy on Incidence of Postoperative Seroma Formation
}

\author{
Erum Najeeb1, Rakhshanda Rashid2 and Shaista Zaffar2
}

\begin{abstract}
Objective: To determine the efficacy of flap fixation technique on formation of postoperative seroma after modified radical mastectomy.

Study Design: Quasi-experimental study.

Place and Duration of Study: General Surgery Department, PIMS Hospital, Islamabad, from August 2014 to February 2015.

Methodology: This study included 70 female patients, aged 16 to 70 years, undergoing modified radical mastectomy, randomly divided into two groups of 35 each, Group A (flap fixation) and Group B (non-flap fixation). Flap fixation was done by suturing flaps by absorbable, fine suture to underlying pectoralis fascia to obliterate the dead space. Two closed suction drains were used in both groups. Patients were discharged on the second postoperative day. Patients and their attendants were thoroughly educated about record of drain output. Patients followed in OPD after one week. Drains were removed when fluid output was less than $50 \mathrm{ml} /$ day. After removal of drains, patient were again called for weekly follow-ups in surgical OPD. Formation of seroma was diagnosed clinically as collection of fluid under the mastectomy flaps and axilla seen as fluctuant, non-tender swelling. Data was collected on proforma designed for the study and analysed by SPSS version 20.0.

Results: Flap fixation group had $2(5.7 \%)$ cases of seroma formation while control group had $3(8.6 \%)$ cases of seroma formation. The difference between both groups statistically was insignificant $(p=0.643)$.

Conclusion: Flap fixation technique has no statistically significant effect on reducing frequency of seroma formation in patients undergoing modified radical mastectomy.
\end{abstract}

Key Words: Seroma, Modified radical mastectomy, Flap fixation technique.

\section{INTRODUCTION}

Breast cancer is the commonest female malignancy worldwide and in Pakistan. ${ }^{1}$ Surgical treatment in the form of either breast conserving surgery or modified radical mastectomy (MRM) with axillary clearance remains the standard treatment for operable breast cancers. ${ }^{2}$ In Pakistan, due to multiple factors, patients with breast cancer present in late stages.1,3-5 This trend makes modified radical mastectomy being done more frequently on such patients. Modified radical mastectomy involves removing whole of breast tissue containing the tumor, overlying nipple areola complex. Level 2 axillary dissection is part of modified radical mastectomy. ${ }^{2}$ This surgery results in a large dead space underneath the mastectomy flaps and axilla, thus becoming a potential space for fluid accumulation.

Postoperative complications of breast cancer surgery includes, flap necrosis, wound infection, and seroma

1 Department of General Surgery, Federal General Hospital, Islamabad, Pakistan

2 Department of General Surgery, PIMS Hospital, Islamabad, Pakistan

Correspondence: Dr. Erum Najeeb, Department of General Surgery, Federal General Hospital, Chak Shehzad,

Islamabad, Pakistan

E-mail: erum.najeeb@yahoo.com

Received: March 14, 2018; Accepted: December 04, 2018 formation. Formation of seroma is the most common early complication. Its reported incidence varies between $24.1-27.5 \% .6,7$

Seroma is collection of sterile, clear fluid formed under mastectomy flaps and axilla. It has been attributed to a number of causes like advanced age, obesity, diabetes mellitus, number of involved lymph nodes, extent of axillary dissection, type of primary breast surgery and neoadjuvant treatment. But overall pathogenesis is poorly understood. 6,7

Seroma formation increases morbidity, hospital stay, frequency of hospital visits, start of adjuvant treatment and wound healing due to failure of flap adherence to underlying chest wall. Ideal wound closure should minimise lymph spillage and serum oozing to provide a means of holding skin flaps securely to the chest wall structures, obliterate dead space and allow rapid removal of fluid as it forms. Therefore, different techniques have been employed in the past to reduce its formation. These techniques include: external compression dressings, delayed removal of drains, shoulder exercises, use of sealants and sclerosing agents, decrease use of electrocautery, and obliteration of dead space by flap fixation using sutures or fibrin glue. $8-13$

Controversies still remain to designate a single method to effectively decrease seroma formation. Different 
studies show benefit of one method over the other. A number of studies support the use of flap fixation technique, after primary breast surgery to reduce incidence of seroma and decrease length of hospital stay.9,11,12,14

Many studies have been done in Pakistan regarding incidence of seroma formation after mastectomy. But thorough literature search shows lack of studies regarding efficacy of various methods of decreasing seroma formation in Pakistan. Although such studies have been done in neighbouring countries like India, Iran and China, which support the flap fixation technique.9,11,14

This study was carried out to see the effect of flap fixation technique on common and cumbersome complications of primary breast surgery, i.e. seroma formation. This may help healthcare managers in better management of patients and decrease patients' morbidity after breast surgery.

\section{METHODOLOGY}

This study was conducted in the General Surgery Department, Pakistan Institute of Medical Sciences, Islamabad, from August 2014 to February 2015, after approval from Institutional Review Board and Ethics Committee. Seventy patients meeting the inclusion criteria were enrolled into the study by convenience sampling technique. Written informed consent was taken from all the patients. All female patients age 18-70 years with breast cancer, undergoing modified radical mastectomy and of ASA I and II, were randomly divided into two groups containing 35 patients each. Women undergoing breast conserving surgery, having clotting and immune dysfunction, with uncontrolled diabetes mellitus, hypertension, advanced liver disease, severely obese (BMI $>40 \mathrm{Kg} / \mathrm{m}^{2}$ ), with previous history of axillary dissection, and undergoing immediate reconstructive surgery were excluded from the study.

Group A was labelled flap fixation group and Group B was non-flap fixation group. After completion of modified radical mastectomy, the Group A patients had flaps stitched in multiple rows, $3 \mathrm{~cm}$ apart with absorbable braided suture (vicryl $3 / 0$ ). The stitches were taken from raw areas between the subcutaneous tissues of the skin flaps and the underlying pectoralis fascia at various parts of the flap, wound edges. If pectoral fascia was excised during surgery because of being involved by tumor, the flaps were fixed to underlying pectoralis major muscle. This technique was also used to obliterate dead space in axilla, taking care of important neurovascular bundle in axilla. Group B was taken as control group, whose flaps were dealt routinely. Two closed suction drains were used after surgery, one under flap and other in axilla. Drain output was monitored and patient was discharged after 48 hours. Patient and attendants were counselled for proper record of drain output, and removed when 24 hours output was less than $50 \mathrm{mls}$. Patients were called for follow-up after discharge after 1 week, 2 weeks and 4 weeks. Patients were observed for formation of seroma, which was recognised as non-tender, fluctuant swelling with serous fluid on aspiration. The frequency of seroma was noted in both groups.

Data was collected on proforma designed for the study, and was analysed using SPSS version 20 . Mean \pm SD was calculated for age. Percentages were calculated for grade and stage of disease and seroma formation. Frequencies of seroma formation between two groups were compared using Chi-square test. $P<0.05$ was taken as statistically significant.

\section{RESULTS}

Seventy cases were included in this study with the mean age of $44.51 \pm 9.99$ years. In Group A, the mean age was $46.26 \pm 9.23$ years while the mean age of Group B was $42.77 \pm 10.55$ years.

Fifty-five patients (78.6\%) had tumour stage II and 15 $(21.4 \%)$ had tumour stage III, while there was no case of stage I and IV.

There were $8(11.43 \%)$ patients of tumour grade I, 37 $(52.86 \%)$ had tumour grade II, while $25(35.71 \%)$ had tumour grade III.

Data was stratified for tumour stage and grade. It was revealed that in stage II tumour, $4(7.3 \%)$ out of 55 patients developed seroma. In stage III, $1(6.7 \%)$ out of 15 patients developed seroma. The difference between both stages was insignificant ( $p=0.936$, Table I).

Data was also stratified for tumour grade. In grade I, 2 $(25 \%)$ out of 8 patients had seroma formation. In grade II tumour, $3(8.1 \%)$ out of 37 patients had seroma formation. In grade III tumour, no $(0 \%)$ patient had seroma formation. The differences between all grades were not significant $(p=0.054$, Table II).

Overall, 5 (7.14\%) patients had seroma formation after surgery, while $65(92.86 \%)$ patients did not develop seroma, and their wound was healing properly.

Table I: Comparison of seroma formation in both groups stratified for tumour stage.

\begin{tabular}{l|l|l|l}
\hline \multirow{2}{*}{ Stage } & \multicolumn{2}{|c|}{ Seroma formation } & \multirow{2}{*}{ p-value } \\
\cline { 2 - 3 } & Yes & No & \\
\hline Stage II $(n=55)$ & $4(7.3 \%)$ & $51(92.7 \%)$ & \\
Stage III $(n=15)$ & $1(6.7 \%)$ & $14(93.3 \%)$ & \multirow{2}{*}{0.936} \\
Total & 5 & 65 & \\
\hline
\end{tabular}

Table II: Comparison of seroma formation in both groups stratified for tumour grade.

\begin{tabular}{l|l|l|l}
\hline \multirow{2}{*}{ Stage } & \multicolumn{2}{|c|}{ Seroma formation } & \multirow{2}{*}{ p-value } \\
\cline { 2 - 3 } & \multicolumn{1}{|c|}{ Yes } & No & \\
\hline grade I $(n=8)$ & $2(25 \%)$ & $6(75 \%)$ & \multirow{2}{*}{0.054} \\
grade II $(n=37)$ & $3(8.1 \%)$ & $34(91.9 \%)$ & \\
grade III $(n=25)$ & $0(0 \%)$ & $25(100)$ & \\
Total & 5 & 65 & \\
\hline
\end{tabular}


In flap fixation group, $2(5.7 \%)$ patients had seroma formation, while $33(94.3 \%)$ remaining patients had proper healing of wound and showed no sign of seroma formation. In non-flap fixation group, $3(8.6 \%)$ cases had seroma formation, while $32(91.4 \%)$ remaining patients showed no sign of seroma formation. The frequency of seroma formation between both the groups was statistically not significant $(p=0.643)$.

\section{DISCUSSION}

Seroma formation is the most common complication after breast surgery. It delays patient recovery, causes unpleasant symptoms, predisposes to wound infection and dehiscence, needs frequent hospital visits, and delays the start of adjuvant treatment. Pathogenesis is poorly understood. Seroma is formed by acute inflammatory exudates due to dissection done in surgery and acute phase of wound healing. Moreover, axillary lymphadenectomy damages lymphatics resulting in lymph leakage. Seroma formation is related to patient factors like body mass index and patient weight. ${ }^{8}$

Various methods have been used in different trials, for preventing seroma. The methods used includes prolonged suction drainage, shoulder immobilisation, mechanical closure of dead space either using compression dressing or fixation of flaps to underlying fascia. ${ }^{8-14}$ Moreover, perioperative uses are of transexamic acid, octreotide usage, tissue sealers, fibrin glue, etc. These methods for the prevention and treatment of seroma remain varied and inconclusive. ${ }^{8-14}$

Recently, there have been continuing debate on mechanical closure of dead space and its effect on seroma prevention. The present study focused on effect of flap fixation technique using sutures on formation of seroma. Flap fixation obliterates the dead space between flap and underlying pectoralis fascia, hence early adherence of flap to fascia and thus decrease in seroma formation. In a study conducted by Sakkaray, flap fixation technique reduced the incidence to $10 \%$ as compared to $40 \%$ incidence in non-flap fixation mastectomy. ${ }^{9}$ Sakkary reported that flap fixation resulted in decrease in total amount of seroma fluid drained; whereas our study, however, did not support his study.

In another study, Wolde et al. did flap fixation by quilting the flap. ${ }^{10}$ They concluded that incidence of seroma decreased from $80.5 \%$ to $22.5 \%(p<0.001)$. They also reported statistically significant results in reducing seroma related complications and surgical site infections.

Bastelaar et al. also supported flap fixation as a valuable option in reducing flap fixation after mastectomy. ${ }^{15}$ Similarly, Bastelaar et al. compared the flap fixation done either by sutures or fibrin glue compared to drain only group in another study. They reported significantly reduced seroma formation after mastectomy using flap fixation technique $(p<0.001) .16$ Altundag supported Bastelaar et al. in his research. ${ }^{17}$ Moreover, he also linked formation of lymphedema of arm after mastectomy with seroma formation. He reported that if seroma formation could be reduced effectively by flap fixation techniques using either sutures or tissue glue, it could have implication on formation of lymphedema, too.

Almond et al. concluded breast flap anchoring following primary breast cancer surgery facilitating early hospital discharge and reducing costs but has no effect on overall seroma formation. 18 The present results support Almond et al. study.

Furthermore, Lumachi et al. identified tumour size as a factor affecting seroma development, 12 but the present results are comparable with those of Hashemi et al., ${ }^{19}$ which suggested that size of tumour, patient's age, positive axillary lymph nodes, or number of lymph nodes dissected are not associated with seroma development. This study supported the earlier evidence of no effect of tumour grade or stage on formation of seroma as quoted by Akinci et al. ${ }^{7}$ The overall frequency of seroma was also low $(n=5,7.14 \%)$ in this study as compared to earlier studies done stating frequency of $24.1 \%$ to $27.5 \%$. Moreover, during data collection, it was observed that the total amount of seroma fluid drained in suction bottles was much less in flap fixation group as compared to non-flap fixation group, supporting the research done by Sakkaray.

A review article by van Bastelaar published recently, reviewed 9 articles to see effect of flap fixation on seroma formation. He concluded that mechanical flap fixation seemed to reduce seroma formation and seroma aspiration after mastectomy. He further suggested that due to non-availability of well powered randomised controlled trials, further research is required. 20

To see the validity of flap fixation technique, it is suggested that studies with a larger sample size be conducted, before abandoning this procedure altogether, especially in Pakistan. During the study, it was also observed that total operating time was 20-30 minutes more in flap fixation group. The fact about this procedure also needs discussion in further studies. Moreover, flap fixation resulted in dimples in flaps that were fixed to underlying fascia. This resulted in cosmetically bad results. Therefore, further studies should investigate in detail the effect of flap fixation with sutures on cosmesis, quality of life and cost effectiveness. This technique may prove to be a valuable step in mastectomy surgery, if supported by further studies.

QUISERMAS, the first multicentre randomised trial is under way and is assessing flap fixation by quilting technique. This trial has included a large sample size to assess the outcome. This study is expected to resolve many of the controversies related to seroma formation and its effective preventive measures. ${ }^{21}$ 


\section{CONCLUSION}

There was no significant decrease in frequency of seroma formation by flap fixation after modified radical mastectomy.

\section{REFERENCES}

1. Jemal A, Siegel R, Ward E, Hao Y, Xu J, Murray T, et al. Cancer statistics, 2008. CA Cancer J Clin 2008; 58:71-96.

2. Sainsbury R. The breast. In: Williams SN, Bulstrode CJK, O'Connell PR, Eds. Bailey and Love's, short practice of surgery. 27th ed. London: Hodder Arnold; 2018.p. 876

3. Collective cancer registry report from Dec. 1994 uptil Dec 2012), available at http://www.shaukatkhanum.org.pk/images/ skm_img/downloads/pdf/ccrr-1994-2012.pdf

4. Naeem M, Khan N, Aman Z, Nasir A, Samad A, Khattak A. Pattern of breast cancer: Experience at Lady Reading Hospital, Peshawer. J Ayub Med Coll Abbottabad 2008; 20:22-5

5. Khokher S, Qureshi MU, Riaz M, Akhtar N, Saleem A. Clinicopathologic profile of breast cancer patients in Pakistan: Ten years data of a local cancer hospital. Asian Pacific $J$ Cancer Prev 2012; 13:693-8

6. Vinton AL, Traverso LW, Jolly PC. Wound complication after modified radical mastectomy with tylectomy with axillary node dissection. Am J Surg 1991; 161:584-8.

7. Akinci $M$, Cetin B, Aslan S, Kulacoglu H. Factors affecting seroma formation after mastectomy with full axillary dissection. Acta Chir Belg 2009; 109:481.

8. Srivastava V, Basu S, Shukla VK. Seroma formation after breast cancer surgery: What we have learned in the last two decades. J Breast Cancer 2012; 15:373-80.

9. Sakkary MA. The value of mastectomy flap fixation in reducing fluid drainage and seroma formation in breast cancer patients. World J Surg Onc 2012; 10:8.

10. Ten Wolde B, van den Wildenberg FJ, Keemer-Gels ME, Polat F, Storbbe LJ. Quilting prevents seroma formation following breast cancer surgery: closing the dead space by quilting prevents seroma following lymph node dissection and mastectomy. Ann Surg Oncol 2014; 21:802-7.
11. Seenivasagam RK, Gupta V, Singh G. Prevention of seroma formation after axillary dissection - a comparative randomized clinical trial of three methods. Breast J 2013; 19:478-84.

12. Lumachi $F$, Brandes AA, Burelli $P$, Basso SM, lacobone $M$, Ermani M. Seroma prevention following axillary dissection in patients with breast cancer by using ultrasound scissors: a prospective clinical study. Eur J Surg Oncol 2004; 5:526-30

13. Bonjar MRM, Maghsoudi H, Samnia R, Saleh P, Parsafar F. Efficacy of fibrin glue on seroma formation after breast surgery. Int J Breast Cancer 2012; 2012:6431-2.

14. Gong $\mathrm{Y}, \mathrm{Xu} J$, Shao J, Cheng $\mathrm{H}$, Wu X, Zhao D, et al. Prevention of seroma formation after mastectomy and axillary dissection by lymph vessel ligation and dead space closure: A randomized trial. Am J Surg 2010; 200:352-6.

15. Van Bastelaar J, Beckers A, Snoeijis M, Beets G, Vissers Y. Flap fixation reduces seroma in patients undergoing mastectomy: A significant implication for clinical practice. World J Surg Oncol 2016; 14:66.

16. Van Bastelaar J, Theunissen LLB, Snoeijs MGJ, Beets LG, Visser YLJ. Flap fixation using tissue glue or sutures appears to reduce seroma aspiration after mastectomy for breast cancer. Clin Breast Cancer 2017; 17:316-21.

17. Altungdag K. Flap fixation using tissue glue or sutures after mastectomy might also reduce risk of developing lymphedema in breast cancer. Clin Breast Cancer 2017; 17:316-21.

18. Almond LM, Khodaverdi L, Kumar B, Coveney EC. Flap anchoring following primary breast cancer surgery facilitates early hospital discharge and reduces costs. Breast Care (Basel) 2010; 5:97-101.

19. Hashemi E, Kaviani A, Najafi M, Ebrahimi M, Hooshmand H, Montazeri A. Seroma formation after surgery for breast cancer. World J Surg Oncol 2004; 2:44.

20. van Bastelaar J, van Roozendaal L, Granzier R, Beets G, Vissers $Y$. A systematic review of flap fixation technique in reducing seroma formation and its sequelae after mastectomy. Breast Cancer Res Treat 2018; 167:409-16.

21. Ouldamer L, Bonastre J, Brunet-Houdard S, Body G, Giraudeau B, Caille A. Dead pace closure with quilting sutures verus conventional closure with drainage for the prevention of seroma after mastectomy for breast cancer(QUISERMAS): protocol for a multicentre randomized controlled trial. BMJ Open 2016; 6:e009903. 